\title{
Constructions of the Soluble Potentials for the Nonrelativistic Quantum System by Means of the Heun Functions
}

\author{
Shishan Dong $\mathbb{D},{ }^{1}$ G. Yáñez-Navarro, ${ }^{2}$ M. A. Mercado Sanchez $\mathbb{D}{ }^{3}$, \\ C. Mejía-García $\oplus^{1},{ }^{2}$ Guo-Hua Sun, ${ }^{4}$ and Shi-Hai Dong $\mathbb{1}^{3}$ \\ ${ }^{1}$ Information and Engineering College, Dalian University, Dalian 116622, China \\ ${ }^{2}$ Escuela Superior de Física y Matemáticas, Instituto Politécnico Nacional, UPALM, CDMX 07738, Mexico \\ ${ }^{3}$ Laboratorio de Información Cuántica, CIDETEC, Instituto Politécnico Nacional, UPALM, CDMX 07700, Mexico \\ ${ }^{4}$ Catedrática CONACyT, CIC, Instituto Politécnico Nacional, UPALM, CDMX 07700, Mexico \\ Correspondence should be addressed to Shi-Hai Dong; dongsh2@yahoo.com
}

Received 25 September 2017; Revised 25 April 2018; Accepted 3 May 2018; Published 3 June 2018

Academic Editor: Won Sang Chung

Copyright (c) 2018 Shishan Dong et al. This is an open access article distributed under the Creative Commons Attribution License, which permits unrestricted use, distribution, and reproduction in any medium, provided the original work is properly cited. The publication of this article was funded by SCOAP ${ }^{3}$.

\begin{abstract}
The Schrödinger equation $\psi^{\prime \prime}(x)+\kappa^{2} \psi(x)=0$ where $\kappa^{2}=k^{2}-V(x)$ is rewritten as a more popular form of a second order differential equation by taking a similarity transformation $\psi(z)=\phi(z) u(z)$ with $z=z(x)$. The Schrödinger invariant $I_{S}(x)$ can be calculated directly by the Schwarzian derivative $\{z, x\}$ and the invariant $I(z)$ of the differential equation $u_{z z}+f(z) u_{z}+g(z) u=0$. We find an important relation for a moving particle as $\nabla^{2}=-I_{S}(x)$ and thus explain the reason why the Schrödinger invariant $I_{S}(x)$ keeps constant. As an illustration, we take the typical Heun's differential equation as an object to construct a class of soluble potentials and generalize the previous results by taking different transformation $\rho=z^{\prime}(x)$ as before. We get a more general solution $z(x)$ through integrating $\left(z^{\prime}\right)^{2}=\alpha_{1} z^{2}+\beta_{1} z+\gamma_{1}$ directly and it includes all possibilities for those parameters. Some particular cases are discussed in detail. The results are also compared with those obtained by Bose, Lemieux, Batic, Ishkhanyan, and their coworkers. It should be recognized that a subtle and different choice of the transformation $z(x)$ also related to $\rho$ will lead to difficult connections to the results obtained from other different approaches.
\end{abstract}

\section{Introduction}

The exact solution of the Schrödinger equation with physical potentials has played an important role in quantum mechanics. This is due to the fact that an exact knowledge of the energy spectrum and the wave functions of the onedimensional Schrödinger equation turns out to be very useful in many applied problems. Generally speaking, for a given external field, one of our main tasks is to show how to solve the differential equation through choosing suitable variables and then find its exact solutions expressed by some special functions. Here we focus on how to construct a class of the solvable potentials within the framework of the nonrelativistic Schrödinger equation. Up to now, a lot of similar works have been carried out with this stimulation [1-9]. For example, Lemieux and Bose presented an advanced analysis of the variable transformation and have listed 8 potentials [3] (written in French). Batic, Williams, and Nowakowski have discussed the general potential allowing reduction of the Schrödinger equation to the general Heun's differential equation by an energy-independent transformation. In particular, Theorem 4.1 in [8] gives the most general form of this potential including 15 parameters instead of 14 parameters, which contain 10 parameters $\left(g_{0,1,2,3,4}\right.$ and $\left.f_{0,1,2,3,4}\right)$ and original parameters $\alpha, \beta, \gamma, \delta, \epsilon, q$ involved in the general Heun equation but with a constraint also named as Fuchsian relation (11). In that work [8], they find that their results cover all results obtained by Bose, Lemieux, Natanzon, and Iwata $[1-4,10]$. Iwata studied the soluble potentials in terms of the hypergeometric functions [10]. It is not surprising to see this since the special functions considered by them are only the special cases of the general Heun function. Just 
recently Ishkhanyan and his coauthors worked out a series of significant works by taking $\rho \propto\left(z-a_{1}\right)^{m_{1}}\left(z-a_{2}\right)^{m_{2}}\left(z-a_{3}\right)^{m_{3}}$, where the parameters $a_{1,2,3}$ are three singularity points, to construct the soluble potentials with some constraints on the parameters $-1 \leq m_{1,2,3} \leq 1$ and $1 \leq m_{1}+m_{2}+m_{3} \leq 3$ $[6,7,9]$. By choosing different values of these parameters which satisfy these constraints, some interesting results have been obtained. However, the approaches taken by Natanzon [4], who constructed a class of the soluble potentials related to the hypergeometric functions, and by Bose [1], who discussed the Riemann and Whittaker differential equations, are different from Ishkhanyan et al. In Bose's classical works $[1,3]$ he only studied a few special cases for the differential equation $\left(z^{\prime}\right)^{2}=\alpha_{1} z^{2}+\beta_{1} z+\gamma_{1}$. Its general solutions were not presented at that time due to the limit on the possible computation condition. In this work our aim is to construct the soluble potentials within the framework of the Schrödinger invariant $I_{S}(x)$ through solving the differential equation of the transformation $z(x)$ directly and then to obtain its more general solutions instead of considering several special cases for the parameters $\alpha_{1}, \beta_{1}$, and $\gamma_{1}$.

The rest of this work is organized as follows. In Section 2 we present the Schwarzian derivative $\{z, x\}$ and the invariant $I(z)$ of the differential equation $u_{z z}+f(z) u_{z}+g(z) u=0$ through acting the similarity transformation $\psi(z)=\phi(z) u(z)$ on the Schrödinger equation. In Section 3 as an illustration we take the Heun's differential equation as a typical example but following slight different approaches taken in [6-9]. All soluble potentials are obtained completely in Section 4 . Some concluding remarks are given in Section 5.

\section{Similarity Transformations to the Schrödinger Equation}

As we know, the Schrödinger equation has the form

$$
\frac{d^{2}}{d x^{2}} \psi(x)+\left[k^{2}-V(x)\right] \psi(x)=0
$$

where we call $k^{2}$ an energy term and $V(x)$ an external potential.

Through choosing a similarity transformation $\psi(z)=$ $\phi(z) u(z)$ where $z=z(x)$, we are able to obtain the following differential equation:

$$
\begin{array}{r}
u_{z z}(z)+\left(\frac{\rho_{z}}{\rho}+\frac{2 \phi_{z}}{\phi}\right) u_{z}(z) \\
+\left[\frac{\phi_{z z}}{\phi}+\frac{\rho_{z} \phi_{z}}{\rho \phi}+\frac{k^{2}-V(x)}{\rho^{2}}\right] u(z)=0, \\
\rho(x)=\frac{d z(x)}{d x},
\end{array}
$$

which can be rewritten as

$$
u_{z z}+f(z) u_{z}+g(z) u=0, \quad u_{z z}=\frac{d^{2} u}{d z^{2}}
$$

which implies that

$$
\begin{aligned}
& f(z)=\left(\frac{\rho_{z}}{\rho}+\frac{2 \phi_{z}}{\phi}\right), \\
& g(z)=\frac{\phi_{z z}}{\phi}+\frac{\rho_{z} \phi_{z}}{\rho \phi}+\frac{k^{2}-V(x)}{\rho^{2}} .
\end{aligned}
$$

Integrating the first differential equation allows us to obtain

$$
\phi(z)=\rho^{-1 / 2} e^{1 / 2 \int f(z) d z} .
$$

Substitution of this into the second differential equation of (4) yields

$$
g-\frac{1}{2} f_{z}-\frac{1}{4} f^{2}=-\frac{1}{2}\left(\frac{\rho_{z}}{\rho}\right)_{z}-\frac{1}{4}\left(\frac{\rho_{z}}{\rho}\right)^{2}+\frac{\left(k^{2}-V\right)}{\rho^{2}}
$$

from which we define the expression [11]

$$
I(z)=g-\frac{f_{z}}{2}-\frac{f^{2}}{4}
$$

as the invariant of (3) (such a process is known as the normal form of the equation; equations which have the same normal form are equivalent). Using Schwarzian derivative,

$$
\begin{aligned}
\{z, x\} & =\frac{d^{2} \log z^{\prime}(x)}{d x^{2}}-\frac{1}{2}\left(\frac{d \log z^{\prime}(x)}{d x}\right)^{2} \\
& =\left(\frac{z^{\prime \prime}(x)}{z^{\prime}(x)}\right)^{\prime}-\frac{1}{2}\left[\frac{z^{\prime \prime}(x)}{z^{\prime}(x)}\right]^{2} \\
& =\frac{z^{\prime \prime \prime}(x)}{z^{\prime}(x)}-\frac{3}{2}\left[\frac{z^{\prime \prime}(x)}{z^{\prime}(x)}\right]^{2}=\rho \rho_{z z}-\frac{1}{2} \rho_{z}^{2},
\end{aligned}
$$

where we have used the relation $z^{\prime \prime}(x)=\rho(d / d x)=\rho \rho_{z}$. Consider (7); then we can rearrange (6) as

$$
\rho^{2} I(z)+\frac{1}{2}\{z, x\}=k^{2}-V(x) \equiv I_{S}(x),
$$

where $I_{S}(x)$ is defined as the Schrödinger invariant $[1,4]$. Thus, the problem of the construction of the soluble potentials for the original Schrödinger equation (1) is solvable on the basis of the functions corresponding to a given $I(z)$; $(7)$ becomes a problem of deciding transformation $z(x)$ such that the relation $\rho^{2} I(z)+(1 / 2)\{z, x\}=z^{\prime}(x)^{2} \rho^{2} I(z)+(1 / 2)\{z, x\}=$ $I_{S}(x)$ holds. The Schrödinger invariant $I_{S}(x)$ is thus characterized by two elements, i.e., the $I(z)$ and the Schwarzian derivative $\{z, x\}$, which is directly related to the transformation function $z(x)$. This means that the Schrödinger invariant exists for all nonrelativistic Schrödinger quantum systems. The possibility that the potentials could be soluble or not depends on the second order ordinary differential equations and also on their solutions which can be written out explicitly by some special functions. 


\section{Application to Heun's Differential Equation}

The Heun's differential equation is given by $[8,12-18]$

$$
\begin{aligned}
u_{z z} & +\left(\frac{\gamma}{z}+\frac{\delta}{z-1}+\frac{\epsilon}{z-a}\right) u_{z}+\frac{\alpha \beta z-q}{z(z-1)(z-a)} u \\
& =0
\end{aligned}
$$

where the parameters satisfy the Fuchsian relation

$$
\alpha+\beta+1=\gamma+\delta+\epsilon
$$

For this equation, the Heun Invariant $I_{h}$ can be calculated as

$$
\begin{aligned}
I_{h}(z)= & \frac{\alpha \beta z-q}{z(z-1)(z-a)}-\frac{1}{4}\left(\frac{\gamma}{z}+\frac{\delta}{z-1}+\frac{\epsilon}{z-a}\right)^{2} \\
& +\frac{1}{2}\left(\frac{\gamma}{z^{2}}+\frac{\delta}{(1-z)^{2}}+\frac{\epsilon}{(a-z)^{2}}\right) \\
= & \frac{A z^{4}+B z^{3}+C z^{2}+D z+F}{z^{2}(z-1)^{2}(z-a)^{2}}
\end{aligned}
$$

$$
I(y)=\frac{\lambda_{0}(y-1)^{2}(y-a)^{2}+\lambda_{1} y^{2}(y-a)^{2}+\lambda_{2} y^{2}(y-1)^{2}+\lambda_{3} y^{2}(y-1)(y-a)+\lambda_{4} y(y-1)(y-a)}{4 y^{2}(y-1)^{2}(y-a)^{2}}
$$

The parameters $A, B, C, D, F$ depend on the singularity $a$ and the parameters $\alpha, \beta, \gamma, \delta, \epsilon, q$; i.e.,

$$
\begin{aligned}
A= & \frac{1}{4}[4 \alpha \beta-(\gamma+\delta+\epsilon-2)(\gamma+\delta+\epsilon)], \\
B= & \frac{1}{2}\{-2(a+1) \alpha \beta \\
& +(\gamma+\delta+\epsilon-2)(a \gamma+a \delta+\gamma+\epsilon)-2 q\} \\
C= & \frac{1}{4}\left\{a^{2}[-(\gamma+\delta-2)](\gamma+\delta)\right. \\
& +a[4 \alpha \beta-2 \epsilon(2 \gamma+\delta)-4 \gamma(\gamma+\delta-2)] \\
& +4(a+1) q-(\gamma+\epsilon-2)(\gamma+\epsilon)\}, \\
D= & \frac{1}{2} a[\gamma(a(\gamma+\delta-2)+\gamma+\epsilon-2)-2 q], \\
F= & -\frac{1}{4} a^{2} \gamma(\gamma-2) .
\end{aligned}
$$

Before ending this part, we give a useful remark about the Heun invariant $I_{h}$ (also named as Bose invariant) as studied by Batic and his coworkers Williams and Nowakowski [8]. They expressed it in another way: where the parameters $\lambda_{0,1,2,3,4}$ are related to the parameters $\alpha, \beta, \gamma, \delta, q$ of the Heun's differential equations. This invariant can also be written as $I(y)=P(y) / Q(y)$ [8]. Comparing this with ours, it is not difficult to find that $c_{0}=4 A, c_{1}=4 B, c_{2}=$ $4 C, c_{3}=4 D, c_{4}=4 F$, where the coefficients $A, B, C, D, F$ are given above. It is worth emphasizing that they decomposed Bose invariant $I(y)$ into two parts, i.e., $I(y)=I_{1}(y) k^{2}+I_{0}(y)$ if and only if $P(y)=R(y) k^{2}+S(y)$ where

$$
\begin{aligned}
& R(y)=\sum_{i=0}^{4} a_{i} y^{i}, \\
& S(y)=\sum_{j=0}^{4} b_{j} y^{j} .
\end{aligned}
$$

Thus, the coefficients $c_{0,1,2,3,4}$ might connect the coefficients $a_{0,1,2,3,4}$ and $b_{0,1,2,3,4}$, further directly related to the parameters $\lambda_{0,1,2,3,4}$ involved in (14) [8]. We refer the reader to this work for more information.

\section{Soluble Potentials Constructed by Heun Invariant Transferred to Schrödinger Invariant}

Now, let us determine functions $\rho=z^{\prime}(x)$ that can be used to transform $I_{h}$ to $I_{S}$ in order to calculate the Schwarzian derivative $\{z, x\}$. The form of the present invariant $I_{h}(z)$ given in (12) suggests taking the class of functions defined by (it should be pointed out that present choice is different from previous one $[7,9]$, in which the $\rho \propto\left(z-a_{1}\right)^{m_{1}}\left(z-a_{2}\right)^{m_{2}}(z-$ $\left.a_{3}\right)^{m_{3}}$ is chosen in order to adapt the mathematical character of the Heun invariant (12))

$$
\rho^{2}=z^{\prime}(x)^{2}=\alpha_{1} z(x)^{2}+\beta_{1} z(x)+\gamma_{1}
$$

where $\alpha_{1}, \beta_{1}, \gamma_{1}$ are three arbitrary constants. Such a choice is to make the $\rho^{2} I_{h}(z)(9)$ generate a constant to cancel the energy level term $k^{2}$. Otherwise, the expansion terms for $\rho^{2} I_{h}(z)$ without including a constant will make the energy level term $k^{2}=0$. This means that the particle moves in a free field. In terms of (16), one has

$$
\{z, x\}=-\frac{\alpha_{1}}{2}-\frac{3}{8} \frac{\beta_{1}^{2}-4 \alpha_{1} \gamma_{1}}{\left(\alpha_{1} z^{2}+\beta_{1} z+\gamma_{1}\right)}
$$

where we have used the relation $\{z, x\}=\rho \rho_{z z}-(1 / 2) \rho_{z}^{2}$ and $\rho=z^{\prime}(x)$. 
To solve (16), we attempt to obtain its general solutions with all arbitrary parameters. In this case, one has

$$
\begin{aligned}
& z(x)^{ \pm} \\
& =\frac{e^{\sqrt{\alpha_{1}}\left[-\left(c_{1} \pm x\right)\right]}\left[\beta_{1}^{2}-4 \alpha_{1} \gamma_{1}-2 \beta_{1} e^{\sqrt{\alpha_{1}}\left(c_{1} \pm x\right)}+e^{2 \sqrt{\alpha_{1}}\left(c_{1} \pm x\right)}\right]}{4 \alpha_{1}} \\
& =-\frac{\beta_{1}}{2 \alpha_{1}}+\frac{e^{\mp \sqrt{\alpha_{1}} x}\left(\beta_{1}^{2}-4 \alpha_{1} \gamma_{1}\right)+e^{ \pm \sqrt{\alpha_{1}} x}}{4 \alpha_{1}},
\end{aligned}
$$

where $c_{1}$ is an integral constant and we take $c_{1}=0$ for simplicity.

Let us study this solution in various options for those parameters. If choosing the constants $\alpha_{1}, \beta_{1}, \gamma_{1}$ and the constants of integration suitably, in terms of above results (18) we can obtain their solutions but ignore unimportant integral constants as follows:

(1) When $\beta_{1}=\gamma_{1}=0, \alpha_{1}=4 a^{2}$, we have

$$
z_{1}^{ \pm}(x)=g \exp ( \pm 2 a x), \quad g \in \text { constant. }
$$

(2) When $\alpha_{1}=-\beta_{1}, \gamma_{1}=0, \alpha_{1}=4 a^{2}$, we have

$$
\begin{aligned}
& z_{2}^{a}=\cosh ^{2}(a x), \\
& z_{2}^{b}=-\sinh ^{2}(a x)
\end{aligned}
$$

(3) When $\alpha_{1}=-\beta_{1}, \gamma_{1}=0, \alpha_{1}=-4 b^{2}$, we have

$$
\begin{aligned}
z_{3}^{a} & =\cos ^{2}(b x), \\
z_{3}^{b} & =\sin ^{2}(b x) .
\end{aligned}
$$

(4) When $\alpha_{1}=\gamma_{1}=0, \beta_{1}=4 c$, we have

$$
z_{4}=c x^{2}, \quad c \in \text { constant }
$$

(5) When $\alpha_{1}=\beta_{1}=0, \gamma_{1}=\sigma^{2}$, we have

$$
z_{5}^{ \pm}= \pm \sigma x
$$

It is not difficult to find that cases (2) and (3) can be obtained by considering the relations $\sin (i x)=i \sinh (x)$ and $\cos (i x)=\cosh (x)$ when $2 a$ is replaced by $2 i b$.

On the other hand, it was recalled that $[1,5]$

$$
\left\{z_{t}, x\right\}=\{z, x\}, \quad z_{t} \equiv \frac{A_{1} z+B_{1}}{C_{1} z+D_{1}}
$$

where $A_{1}, B_{1}, C_{1}, D_{1}$ are constants but $A_{1} D_{1}-B_{1} C_{1} \neq 0$. From (24), we have

$$
z=\frac{D_{1} z_{t}-B_{1}}{A_{1}-C_{1} z_{t}} .
$$

Differentiating $z_{t}$ given in (24) with respect to $x$ and eliminating the variable $z$, one has

$$
\begin{aligned}
z_{t}^{\prime}(x) & =\frac{d z_{t}}{d x}=-\frac{\left(C_{1} z_{t}-A_{1}\right)^{2}}{B_{1} C_{1}-A_{1} D_{1}} z^{\prime}(x) \\
\left(\frac{d z_{t}}{d x}\right)^{2} & =\frac{\left(C_{1} z_{t}-A_{1}\right)^{2}}{\left(B_{1} C_{1}-A_{1} D_{1}\right)^{2}}\left[\alpha_{1}\left(B_{1}-D_{1} z_{t}\right)^{2}+\beta_{1}\left(B_{1}-D_{1} z_{t}\right)\left(C_{1} z_{t}-A_{1}\right)+\gamma_{1}\left(C_{1} z_{t}-A_{1}\right)^{2}\right] \\
& =\frac{\left(A_{1}-C_{1} z_{t}\right)^{2}\left(D_{1} z_{t}-B\right)\left[\beta_{1}\left(A_{1}-C_{1} z_{t}\right)-\alpha_{1} B_{1}+\alpha_{1} D_{1} z_{t}\right]+\gamma_{1}\left(A_{1}-C_{1} z_{t}\right)^{4}}{\left(B_{1} C_{1}-A_{1} D_{1}\right)^{2}}
\end{aligned}
$$

where $z^{\prime}(x)$ is given by (16). It is not difficult to see that the solutions of (26) are also possible transformations since it is a generalization of (16). Up to now, we have found a class of functions for transforming $I_{h}$ to $I_{S}$. It is worth noting that this class of functions can be characterized differently. We are going to give a useful remark on the $z_{t}^{\prime}(x)$ given in (26). If we use this to calculate the Schrödinger invariant $I_{S}(x)(9)$, then we will find that the soluble potentials would become rather complicated. Therefore, we do not consider this for simplicity, but it should be recognized that the variable $z_{t}$ is just $z(x)$ as given in (16).

We are now in the position to construct the simple Schrödinger invariant corresponding to the invariant of the general Heun's differential equation with the aid of the Schwarzian derivative (17) we obtained above. First, let us consider the simpler transform (16). Substituting (12) and (17) into (9) allows us to obtain the following useful Schrödinger invariant:

$$
\begin{aligned}
I_{S} & =\rho^{2} I_{h}+\frac{1}{2}\{z, x\}=\left(\alpha_{1} z^{2}+\beta_{1} z+\gamma_{1}\right) \\
& \cdot\left\{\frac{\alpha \beta z-q}{z(z-1)(z-a)}-\frac{1}{4}\left(\frac{\gamma}{z}+\frac{\delta}{z-1}+\frac{\epsilon}{z-a}\right)^{2}\right. \\
& \left.+\frac{1}{2}\left[\frac{\gamma}{z^{2}}+\frac{\delta}{(z-1)^{2}}+\frac{\epsilon}{(z-a)^{2}}\right]\right\}-\left(\frac{\alpha_{1}}{4}\right. \\
& \left.+\frac{3}{16} \frac{\beta_{1}^{2}-4 \alpha_{1} \gamma_{1}}{\alpha_{1} z^{2}+\beta_{1} z+\gamma_{1}}\right)=\left(\alpha_{1} z^{2}+\beta_{1} z+\gamma_{1}\right) \\
& \cdot\left[\frac{A z^{4}+B z^{3}+C z^{2}+D z+F}{z^{2}(z-1)^{2}(z-a)^{2}}\right]-\left(\frac{\alpha_{1}}{4}\right. \\
& \left.+\frac{3}{16} \frac{\beta_{1}^{2}-4 \alpha_{1} \gamma_{1}}{\alpha_{1} z^{2}+\beta_{1} z+\gamma_{1}}\right),
\end{aligned}
$$

where we have used relation (12). 
Let us write down the Schrödinger invariants (essentially related to potentials) based on (19), (20), (21), (22), and (23).

(i)

$$
\begin{aligned}
& I_{S}\left(H 1^{+}\right) \\
& \quad=a^{2}\left\{\frac{4\left[g e^{2 a x}\left(g e^{2 a x}\left(g e^{2 a x}\left(A g e^{2 a x}+B\right)+C\right)+D\right)+F\right]}{\left(a-g e^{2 a x}\right)^{2}\left(g e^{2 a x}-1\right)^{2}}\right. \\
& \quad-1\}, \\
& I_{S}\left(H 1^{-}\right) \\
& \quad=a^{2}\left\{\frac{4\left[B g^{3} e^{2 a x}+C g^{2} e^{4 a x}+D g e^{6 a x}+F e^{8 a x}+A g^{4}\right]}{\left(e^{2 a x}-g\right)^{2}\left(g-a e^{2 a x}\right)^{2}}\right. \\
& \quad-1\} .
\end{aligned}
$$

(ii)

$$
\begin{aligned}
& I_{S}\left(H 2^{a}\right)=-\frac{a^{2} \operatorname{csch}^{2}(2 a x)}{\left[\cosh ^{2}(a x)-a\right]^{2}}\left\{3 a^{2}+(4-16 A)\right. \\
& \cdot \cosh ^{8}(a x)-4(2 a+4 B+1) \cosh ^{6}(a x) \\
& \quad+[4 a(a+2)-16 C+3] \cosh ^{4}(a x) \\
& \left.\quad-2[a(2 a+3)+8 D] \cosh ^{2}(a x)-16 F\right\}, \\
& I_{S}\left(H 2^{b}\right)=-\frac{a^{2} \sinh ^{4}(a x) \tanh ^{2}(a x)}{4\left[\sinh ^{2}(a x)+a\right]^{2}}\left\{\left(3 a^{2}-16 F\right)\right. \\
& \quad \cdot \operatorname{csch}^{8}(a x)+4(2 a+4 B+1) \operatorname{csch}^{2}(a x) \\
& \quad+[4 a(a+2)-16 C+3] \operatorname{csch}^{4}(a x) \\
& \left.\quad+2[a(2 a+3)+8 D] \operatorname{csch}^{6}(a x)-16 A+4\right\} .
\end{aligned}
$$

(iii)

$$
\begin{aligned}
I_{S} & \left(H 3^{a}\left\{3^{b}\right\}\right)=-\frac{b^{2} \csc ^{2}(2 b x)}{\left[\cos ^{2}(b x)\left\{\sin ^{2}(b x)\right\}-a\right]^{2}}\left\{3 a^{2}\right. \\
& +(4-16 A) \cos ^{8}(b x)\left\{\sin ^{8}(b x)\right\} \\
& -4(2 a+4 B+1) \cos ^{6}(b x)\left\{\sin ^{6}(b x)\right\} \\
& +[4 a(a+2)-16 C+3] \cos ^{4}(b x)\left\{\sin ^{4}(b x)\right\} \\
& \left.-2[a(2 a+3)+8 D] \cos ^{2}(b x)\left\{\sin ^{2}(b x)\right\}-16 F\right\} .
\end{aligned}
$$

(iv)

$$
I_{S}(H 4)
$$

$$
\begin{aligned}
= & \frac{16\left\{c x^{2}\left[c x^{2}\left(c x^{2}\left(A c x^{2}+B\right)+C\right)+D\right]+F\right\}}{4 x^{2}\left(c x^{2}-1\right)^{2}\left(a-c x^{2}\right)^{2}} \\
& -\frac{3}{4 x^{2}} .
\end{aligned}
$$

(v)

$$
I_{S}\left(H 5^{ \pm}\right)=\frac{\sigma x[\sigma x(\sigma x(A \sigma x \pm B)+C) \pm D]+F}{x^{2}(\sigma x \mp 1)^{2}(a \mp \sigma x)^{2}} .
$$

Here, we have used the symbol $\left(\mathrm{Hn}^{( \pm, a, b)}\right)$ to denote the above invariants, $H$ referring to $I_{h}$, and $n^{( \pm, a, b)}$ to $z_{n}^{( \pm, a, b)}$ and $\{$, referring to results for $\left\{3^{b}\right\}$. Let us analyze these potentials through expanding them as follows.

For the (i) case, we have

$$
\begin{aligned}
V_{I_{S}}\left(H 1^{+}\right)= & a^{2}(4 A-1)+\frac{A_{1}^{+}}{\left(g e^{2 a x}-1\right)^{2}} \\
& +\frac{B_{1}^{+}}{\left(g e^{2 a x}-1\right)}+\frac{C_{1}^{+}}{\left(g e^{2 a x}-a\right)} \\
& +\frac{D_{1}^{+}}{\left(g e^{2 a x}-a\right)^{2}}
\end{aligned}
$$

where

$$
\begin{aligned}
& A_{1}^{+}=\frac{4\left(a^{2} A+a^{2} B+a^{2} C+a^{2} D+a^{2} F\right)}{(a-1)^{2}}, \\
& B_{1}^{+} \\
& =\frac{4\left(4 a^{3} A+3 a^{3} B+2 a^{3} C+a^{3} D-2 a^{2} A-a^{2} B+a^{2} D+2 a^{2} F\right)}{(a-1)^{3}}, \\
& C_{1}^{+} \\
& =\frac{4\left(2 a^{6} A-4 a^{5} A+a^{5} B-3 a^{4} B-2 a^{3} C-a^{3} D-a^{2} D-2 a^{2} F\right)}{(a-1)^{3}}, \\
& D_{1}^{+}=\frac{4\left(a^{6} A+a^{5} B+a^{4} C+a^{3} D+a^{2} F\right)}{(a-1)^{2}} .
\end{aligned}
$$

For case (ii), we have

$$
\begin{aligned}
V_{I_{S}}\left(H 2^{a}\right)= & \frac{A_{2}^{a} \operatorname{csch}^{2}(2 a x)}{\left[\cosh ^{2}(a x)-a\right]^{2}}+\frac{B_{2}^{a} \operatorname{csch}^{2}(a x)}{\left[\cosh ^{2}(a x)-a\right]^{2}} \\
& -\frac{C_{2}^{a} \operatorname{coth}^{2}(a x)}{\left[\cosh ^{2}(a x)-a\right]^{2}} \\
& +\frac{D_{2}^{a} \cosh ^{2}(a x) \operatorname{coth}^{2}(a x)}{\left[\cosh ^{2}(a x)-a\right]^{2}} \\
& +\frac{E_{2}^{a} \cosh ^{4}(a x) \operatorname{coth}^{2}(a x)}{\left[\cosh ^{2}(a x)-a\right]^{2}}
\end{aligned}
$$


where

$$
\begin{aligned}
& A_{2}^{a}=a^{2}\left(16 F-3 a^{2}\right), \\
& B_{2}^{a}=\frac{a^{2}[a(2 a+3)+8 D]}{2}, \\
& C_{2}^{a}=\frac{a^{2}[4 a(a+2)-16 C+3]}{4}, \\
& D_{2}^{a}=a^{2}(2 a+4 B+1), \\
& E_{2}^{a}=a^{2}(4 A-1) .
\end{aligned}
$$

For case (iii), one has

$$
\begin{aligned}
V_{I_{S}}\left(H 3^{a}\right)= & \frac{A_{3}^{a} \csc ^{2}(2 b x)}{\left[\cos (b x)^{2}-a\right]^{2}}+\frac{B_{3}^{a} \csc ^{2}(b x)}{\left[\cos (b x)^{2}-a\right]^{2}} \\
& -\frac{C_{3}^{a} \cot ^{2}(b x)}{\left[\cos (b x)^{2}-a\right]^{2}} \\
& +\frac{D_{3}^{a} \cos ^{2}(b x) \cot ^{2}(b x)}{\left[\cos (b x)^{2}-a\right]^{2}} \\
& +\frac{E_{3}^{a} \cos { }^{4}(b x) \cot ^{2}(b x)}{\left[\cos (b x)^{2}-a\right]^{2}}
\end{aligned}
$$

where

$$
\begin{aligned}
& A_{3}^{a}=b^{2}\left(16 F-3 a^{2}\right), \\
& B_{3}^{a}=\frac{b^{2}[a(2 a+3)+8 D]}{2}, \\
& C_{3}^{a}=\frac{b^{2}[4 a(2+a)-16 C+3]}{4}, \\
& D_{3}^{a}=b^{2}(2 a+4 B+1), \\
& E_{3}^{a}=b^{2}(4 A-1) .
\end{aligned}
$$

For the special case (iv), we have

$$
\begin{aligned}
V_{I_{S}}(H 4)= & \frac{A_{4}}{x^{2}}+\frac{B_{4}}{\left(c x^{2}-a\right)^{2}}+\frac{C_{4}}{\left(c x^{2}-a\right)} \\
& +\frac{D_{4}}{\left(c x^{2}-1\right)^{2}}+\frac{E_{4}}{\left(c x^{2}-1\right)}
\end{aligned}
$$

where

$$
\begin{aligned}
& A_{4}=\frac{16 F-3 a^{2}}{4 a^{2}}, \\
& B_{4}=\frac{4 c\left(a^{4} A+a^{3} B+a^{2} C+a D+F\right)}{(a-1)^{2} a}, \\
& C_{4} \\
& =\frac{4 c\left(a^{5} A-3 a^{4} A-2 a^{3} B-a^{3} C-a^{2} C-2 a^{2} D-3 a F+F\right)}{(a-1)^{3} a^{2}}, \\
& D_{4}=\frac{4 c(A+B+C+D+F)}{(a-1)^{2}}, \\
& E_{4}=\frac{4 c(3 a A+2 a B+a C-a F-A+C+2 D+3 F)}{(a-1)^{3}} .
\end{aligned}
$$

For case (v), one has

$$
\begin{aligned}
V_{I_{S}}\left(H 5^{-}\right)= & \frac{A_{3}^{-}}{x}+\frac{B_{3}^{-}}{x^{2}}+\frac{C_{3}^{-}}{(a+\sigma x)^{2}}+\frac{D_{3}^{-}}{(a+\sigma x)} \\
& +\frac{E_{3}^{-}}{(\sigma x+1)^{2}}+\frac{F_{3}^{-}}{(\sigma x+1)}
\end{aligned}
$$

where

$$
\begin{aligned}
& A_{5}^{-}=\frac{-\sigma(a D+2 a F+2 F)}{a^{3}}, \\
& B_{3}^{-}=\frac{F}{a^{2}}, \\
& C_{5}^{-}=\frac{\sigma^{2}\left(a^{4} A+a^{3} B+a^{2} C+a D+F\right)}{(a-1)^{2} a^{2}}, \\
& D_{5}^{-} \\
& =\frac{\sigma^{2}\left(2 a^{4} A+a^{4} B+a^{3} B+2 a^{3} C+3 a^{2} D-a D+4 a F-2 F\right)}{(a-1)^{3} a^{3}}, \\
& E_{5}^{-}=\frac{\sigma^{2}(A+B+C+D+F)}{(a-1)^{2}}, \\
& F_{5}^{-}=\frac{\sigma^{2}(-2 a A-a B+a D+2 a F-B-2 C-3 D-4 F)}{(a-1)^{3}} .
\end{aligned}
$$

Obviously, the potential given in case (i) is more complicated than the usual Eckart potential. The potentials discussed in cases (ii) and (iii) are more complicated than the first and second type Pöschl-Teller potentials. The potential studied in case (iv) is more like the $x^{-2}+x^{-4}$ while the potential given in case (v) essentially is the sum of the Coulomb potential plus a centrifugal term. The other cases such as $V_{I_{S}}\left(H 1^{-}\right), V_{I_{S}}\left(H 2^{b}\right), V_{I_{S}}\left(H 3^{b}\right), V_{I_{S}}\left(H 5^{+}\right)$have similar properties to their parters. Now, we give a useful remark on connections between present results and previous ones obtained. Even though Lemieux and Bose have also studied the $\cosh ^{2}(a x)$ transformation, the soluble potential constructed by them is different from the present expression (35) [see Table 1 from Lemieux et al. (1969)]. The parameters involved in (35) such as $A_{2}^{a}, B_{2}^{a}, C_{2}^{a}, D_{2}^{a}$, and $E_{2}^{a}$ connect 
directly with those parameters $A, B, C, D$, and $F$ as given in (13), which are further related to the parameters of the Heun's differential equation (10). The connection between (35) and the potential through taking the transformation $z=\cosh ^{2}(\alpha x)$ studied by Lemieux and Bose [3] in Table 1 cannot be found easily and directly even though we consider the relation $\cosh ^{2}(x)-\sinh ^{2}(x)=1$. This is because the parameter $a$ taken here is a singular point as shown in (10). The similar case is also for (19) and (33), in which the parameters $A, A_{1}^{+}, B_{1}^{+}, C_{1}^{+}, D_{1}^{+}$also depend on the parameters $A, B, C, D, F$. Likewise, we cannot find direct connections between (33) and that given in [3], in which $z=\left(d+g e^{\alpha x}\right)$ is taken. On the other hand, it is seen that the Heun differential equation in present study is special case of the general Heun equation [9], in which we take $a_{1}=0, a_{2}=1$, and $a_{3}=a$. The transformation $z_{1}^{ \pm}=g \exp ( \pm 2 a x)$ given in (19) and the potential invariant (33) are similar to those given in the 9th line of Table 1 in [9] [see Table 1 from Ishkhanyan (2018)]. The transformations (20) and (21) as well as the potentials (35) and (37) derived from them are similar to the 11th case of Table 3 [see Table 3 from Ishkhanyan (2018)], but the direct connections between them are also very difficult.

Now, let us study the wave function. In terms of (5) and the function

$$
f(z)=\left(\frac{\gamma}{z}+\frac{\delta}{z-1}+\frac{\epsilon}{z-a}\right)
$$

given in (10), one has the following form:

$$
\phi(z)=\frac{1}{\sqrt{\rho}} z^{\gamma / 2}(z-1)^{\delta / 2}(z-a)^{\epsilon / 2},
$$

where $\rho$ given in (16) depends on the solutions (18), while those particular cases are given in (19) to (23). The partial wave function $u(x)$ involved in the whole wave function $\psi(z)=\phi(z) u(z)$ is given by the Heun functions $H_{l}(a, q, \alpha, \beta, \gamma, \delta, \epsilon ; z)$. Thus, the wave function is given by

$$
\begin{aligned}
& \psi(z)=\phi(z) u(z)=\frac{1}{\sqrt{\rho}} z^{\gamma / 2}(z-1)^{\delta / 2}(z-a)^{\epsilon / 2} \\
& \cdot H_{l}(a, q, \alpha, \beta, \gamma, \delta, \epsilon ; z) .
\end{aligned}
$$

\section{Conclusion}

The Schrödinger equation is rewritten as a more popular form of a second order differential equation through taking a similarity transformation. We find that this classical equation is closely related to the Schwarzian derivative and the invariant identity of the differential equation $u_{z z}+f(z) u_{z}+$ $g(z) u=0$. As a typical differential equation, the corresponding mathematical properties of the Heun's differential equation are studied. Before ending this work, we give a few useful remarks. First, let us consider the Schrödinger equation (1) and (9). We find that the Schrödinger equation can also be rewritten as $\nabla^{2} \psi(x)=-I_{S}(x) \psi(x)$. Since $\nabla^{2}$ represents the kinetic term $T$ of the moving particle, it should keep invariant for the same particle. This is also reflection of the conservation of energy $T+V=E$. Second, it should be pointed out that Batic et al. also presented the most general potential $V_{H}(x)$ in their significant work [8] with the following form:

$$
\begin{aligned}
& V_{H}(x)=\frac{y^{2}(y-1)^{2}(y-a)^{2}}{R^{2}(y)}\left[\frac{d^{2} R(y)}{d y^{2}}\right. \\
& +\frac{G(y) d R(y) / d y-2 R(y) d G(y) / d y}{y(y-1)(y-a)} \\
& \left.+\frac{R(y) G^{2}(y)}{y^{2}(y-1)^{2}(y-a)^{2}}-\frac{5(d R(y) / d y)^{2}}{4 R(y)}\right] \\
& -\frac{S(y)}{R(y)}
\end{aligned}
$$

where

$$
\begin{aligned}
G(y)= & 3 y^{2}-2(a+1) y+a, \\
R(y)= & g_{0}(y-1)^{2}(y-a)^{2}+g_{1} y^{2}(y-a)^{2} \\
& +g_{2} y^{2}(y-1)^{2}+g_{3} y^{2}(y-1)(y-a) \\
& +g_{4} y(y-1)(y-1), \\
S(y)= & f_{0}(y-1)^{2}(y-a)^{2}+f_{1} y^{2}(y-a)^{2} \\
& +f_{2} y^{2}(y-1)^{2}+f_{3} y^{2}(y-1)(y-a) \\
& +f_{4} y(y-1)(y-1) .
\end{aligned}
$$

The coordinate transformation $y=y(x)$ satisfies the differential equation

$$
R(y)\left(y^{\prime}\right)^{2}=4 y^{2}(y-1)^{2}(y-a)^{2},
$$

from which we find that $\left(y^{\prime}\right)^{2} \propto z^{\prime}(x)^{2}$ as given in (16). The unique differential equation (16) will make us obtain the exact solution of $z(x)$ as shown in (18), from which we have all possible solutions through choosing the different values of those parameters $\alpha_{1}, \beta_{1}$, and $\gamma_{1}$. The expressions for these parameters were not given explicitly in [8]. Third, the results obtained from slightly different calculation process would be very difficult to be connected to each other. The main difference is from the choice of the transformation function $z(x)$, i.e., the $z^{\prime}(x)$ taken in [9] (see equations (11) and (13) of [9]) is different from that of present work (16). Fourth, the present study cannot be copied to the Klein-Gordon equation case since the latter case is given by $\left(-\nabla^{2}+M^{2}\right) \psi(x)=$ $(E-V(x))^{2} \psi(x)$. The complications are from the terms $2 E V(x), E^{2}$, and $V(x)^{2}$ when it is expanded, in particular the mixture term $2 E V(x)$. This is absolutely different from the Schrödinger equation case. Fifth, the potentials obtained by these approaches are most general, but they are not practical since they include too many parameters. We will find that it is very difficult for these parameters to find direct connections with those physical or chemical parameters. This is the reason 
why the potential parameters are relatively few for those wellknown physical and chemical potentials. Nevertheless, the potentials obtained by this way will provide a theoretical guidance to the physical or chemical potential models.

\section{Conflicts of Interest}

The authors declare that there are no conflicts of interest regarding the publication of this paper.

\section{Acknowledgments}

This work is supported partially by Project 20170938-SIPIPN, COFAA-IPN, Mexico, and CONACYT Grant no. 288856-CB-2016, Mexico.

\section{References}

[1] A. K. Bose, "Solvable potentials," Physics Letters B, vol. 7, pp. 245246, 1963.

[2] A. K. Bose, "A class of solvable potentials," Il Nuovo Cimento, vol. 32, no. 3, pp. 679-688, 1964.

[3] A. Lemieux, A. K. Bose, and Ann. Inst, "Annales de l'Institut Henri Poincaré C," Analyse Non Linéaire, vol. 10, p. 259, 1969.

[4] G. A. Natanzon, "General properties of potentials for which the Schrödinger equation can be solved by means of hypergeometric functions," Theoretical and Mathematical Physics, vol. 38, p. 146, 1979.

[5] M. J. Richardson, "Approximate Solutions to the OneDimensional Schrödinger Equation by the Method of Comparison Equations," Physical Review A: Atomic, Molecular and Optical Physics, vol. 8, no. 2, p. 781, 1973.

[6] A. Ishkhanyan and V. Krainov, "A conditionally integrable Schrödinger potential of a bi-confluent Heun class," Journal of Physics: Conference Series, vol. 131, p. 342, 2016.

[7] A. M. Ishkhanyan, "Potentials for which the Schrödinger equation is solvable in terms of the confluent Heun functions," Teoreticheskaya i Matematicheskaya Fizika, vol. 188, no. 1, pp. 20-35, 2016.

[8] D. Batic, R. Williams, and M. Nowakowski, "Potentials of the Heun class," Journal of Physics A: Mathematical and General, vol. 46, no. 24, 245204, 21 pages, 2013.

[9] A. M. Ishkhanyan, "Schrödinger potentials solvable in terms of the general Heun functions," Annals of Physics, vol. 388, pp. 456471, 2018.

[10] G. Iwata, "Nat. Sci. Rep," Ochanomizu Univ, vol. 25, p. 25, 1971.

[11] E. L. Ince, Ordinary Differential Equations, Dover Publications, New York, NY, USA, 1956.

[12] C. A. Downing, "On a solution of the Schrödinger equation with a hyperbolic double-well potential," Journal of Mathematical Physics, vol. 54, no. 7, 072101, 8 pages, 2013.

[13] R. R. Hartmann, "Bound states in a hyperbolic asymmetric double-well," Journal of Mathematical Physics, vol. 55, no. 1, 012105, 6 pages, 2014.

[14] A. Ronveaux, Heuns Differential Equations, Oxford Press, 1995.

[15] D. Agboola, "On the solvability of the generalized hyperbolic double-well models," Journal of Mathematical Physics, vol. 55, no. 5, 052102, 8 pages, 2014.
[16] G. H. Sun, S. H. Dong, T. Dytrych, K. D. Launey, and J. P. Draayer, "Quantum information entropy for a hyperbolical potential function," International Journal of Quantum Chemistry, vol. 115, p. 891, 2015.

[17] S. Dong, Q. Fang, B. J. Falaye, G. Sun, C. Yáñez-Márquez, and S. Dong, "Exact solutions to solitonic profile mass Schrödinger problem with a modified Pöschl-Teller potential," Modern Physics Letters A, vol. 31, no. 04, p. 1650017, 2016.

[18] S. Dong, G. H. Sun, B. J. Falaye, and S. H. Dong, "Semi-exact solutions to position-dependent mass Schrödinger problem with a class of hyperbolic potential V0tanh(ax)," The European Physical Journal Plus, vol. 131, no. 5, p. 176, 2016. 

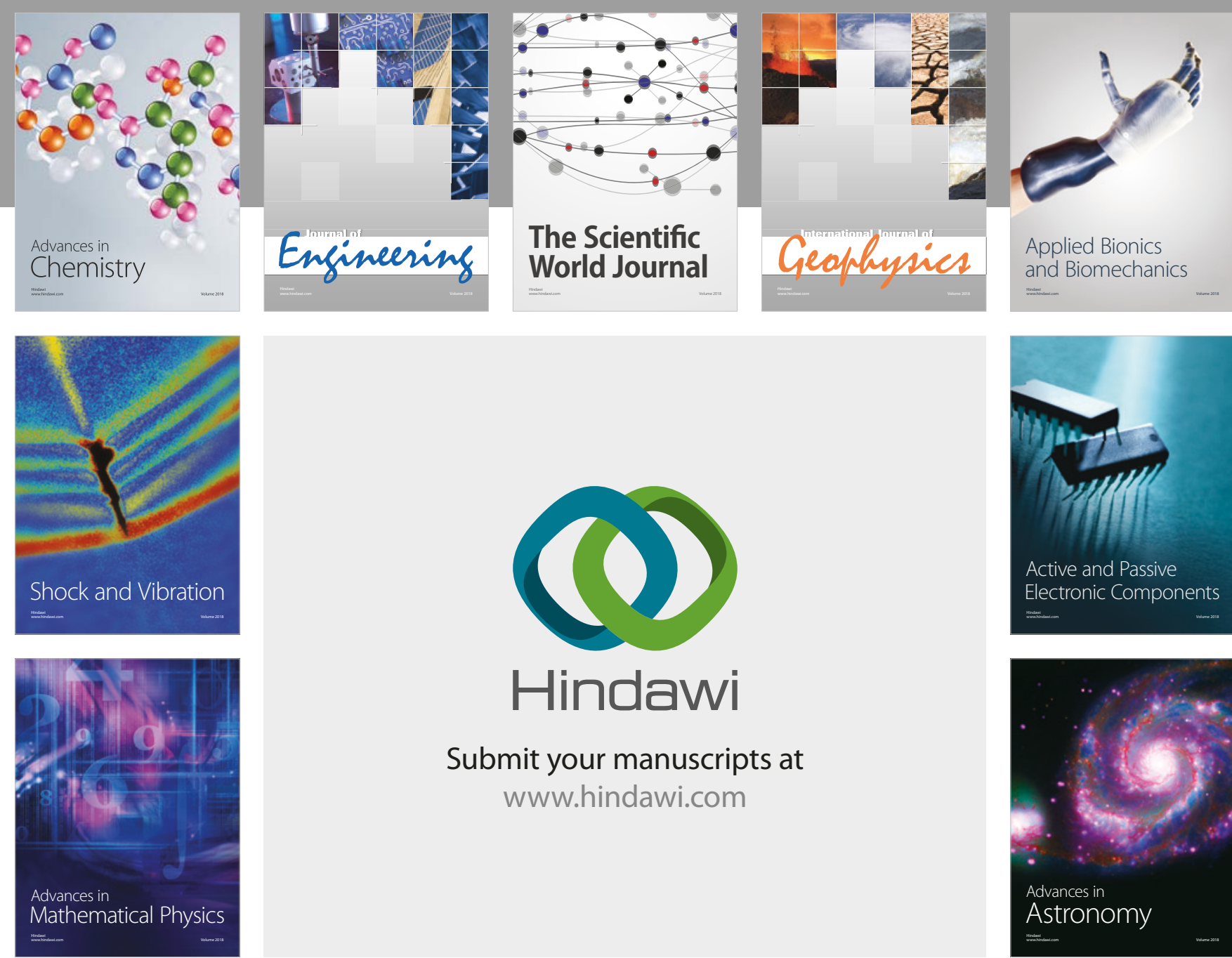

Submit your manuscripts at

www.hindawi.com

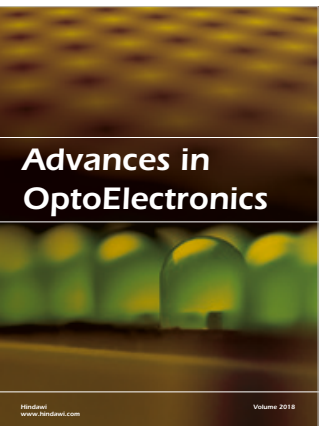

\section{Rotcting Machinery}
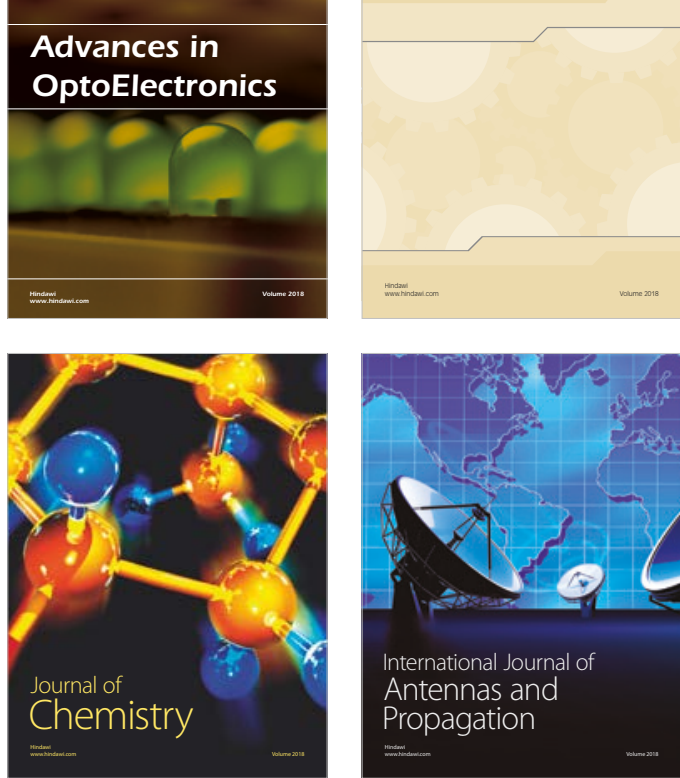

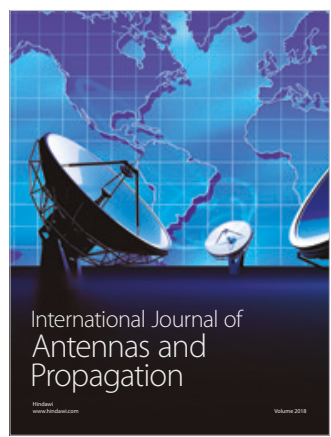

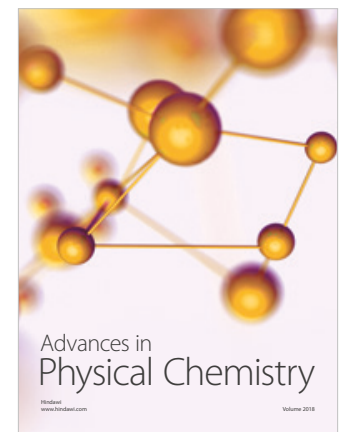

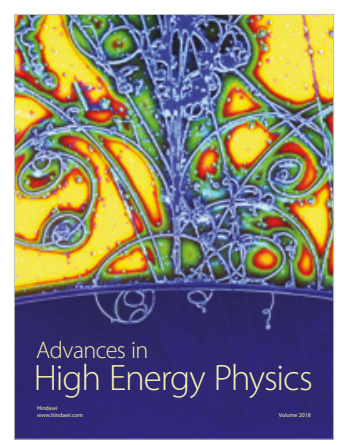

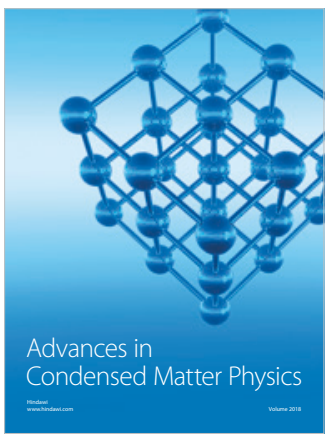

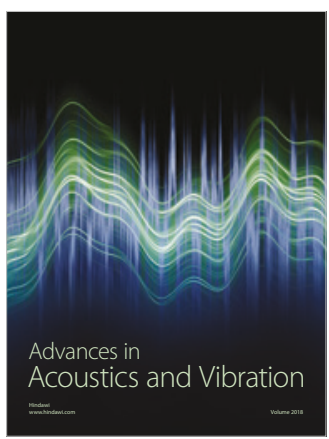

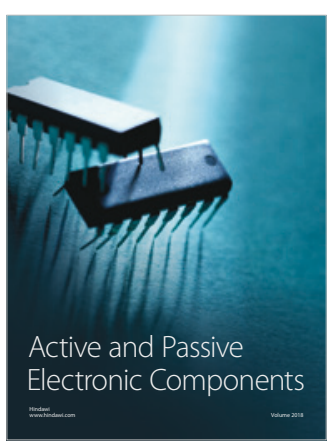
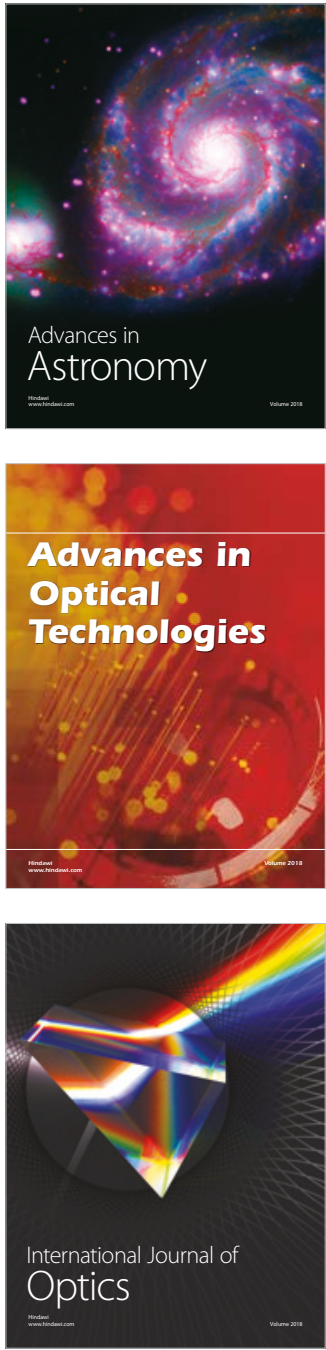